\title{
Strength training with repetitions to failure does not provide additional strength and muscle hypertrophy gains in young women
}

\author{
Saulo Martorelli (1), Eduardo Lusa Cadore (2), Mikel Izquierdo (3), Rodrigo Celes \\ (1), André Martorelli (1,4), Vitor Alonso Cleto (1), José Gustavo Alvarenga (1), \\ Martim Bottaro (1)
}

(1) College of Physical Education, University of Brasilia, Brasilia, DF, Brazil; (2) School of Physical Education, Federal University of Rio Grande do Sul, Porto Alegre, RS, Brazil; (3) Department of Health Sciences, Public University of Navarre, Campus de Tudela, Tudela, Navarre, Spain; (4) Federal Institute of Goias (IFG), Valparaiso, GO, Brazil.

This article is distributed under the terms of the Creative Commons Attribution Noncommercial License (CC BY-NC 4.0) which permits any noncommercial use, distribution, and reproduction in any medium, provided the original author(s) and source are credited.

\begin{abstract}
This study investigated the effects of a 10-week resistance training to failure on neuromuscular adaptations in young women. Eighty-nine active young women were randomly assigned to one of three groups: 1) repetitions to failure (RF; three sets of repetitions to failure); 2) repetitions not to failure with equalized volume (RNFV; four sets of 7 repetitions); and 3) repetitions not to failure (RNF; three sets of 7 repetitions). All groups performed the elbow flexor exercise (bilateral biceps curl) and trained 2 days per week using $70 \%$ of 1RM. There were significant increases $(\mathrm{p}<0.05)$ in muscle strength after $5(15.9 \%$ for RF, $18.4 \%$ for RNF, and $19.9 \%$ for RNFV) and 10 (28.3\% for RF, $26.8 \%$ for RNF, and $28.3 \%$ for RNFV) weeks of training, with no significant differences between groups. Additionally, muscular endurance increased after 5 and 10 weeks, with no differences between groups. However, peak torque (PT) increased significantly at $180^{\circ} . \mathrm{s}-1$ in the RNFV $(13.7 \%)$ and RNF $(4.1 \%)$ groups ( $\left.\mathrm{p}<0.05\right)$, whereas no changes were observed in the RF group (-0.5\%). Muscle thickness increased significantly $(\mathrm{p}<0.05)$ in the RF and RNFV groups after 5 (RF: $8.4 \%$ and RNFV: $2.3 \%$ ) and 10 weeks of training (RF: $17.5 \%$, and RNFV: $8.5 \%$ ), whereas no significant changes were observed in the RNF group (3.9 and $2.1 \%$ after 5 and 10 weeks, respectively). These data suggest that shortterm training of repetitions to failure do not yield additional overall neuromuscular improvements in young women.
\end{abstract}

Key Words: Strength training to failure, muscle hypertrophy, young women, fatigue, maximum exercise.

Eur J Transl Myol 27 (2): 113-120

Resistance training intensity is a key factor to maximize training adaptations, and it is usually determined by the percentage of the one repetition maximum (1RM) or the number of repetitions that one may perform in relation to the number of maximum repetitions until concentric failure (i.e six repetitions with a 12 RM loading). Some authors have suggested that the number of repetitions performed until concentric failure during strength training (i.e., the inability to complete a repetition in a full range of motion at a specific overload ${ }^{1-4}$ may be a strategy to increase the motor unit activation and mechanical stress. $^{5-7}$ On the other hand, the number of training sets performed until concentric failure have been associated with decreases in total work and muscle power along with greater muscle cell metabolism disturbances and neuromuscular fatigue ${ }^{8}$. Therefore, the possible physiological mechanism that support the performance of repetitions until to failure is quite controversial. In fact, the results regarding the effectiveness of training until concentric failure to improve neuromuscular adaptations is still controversial. Although some authors have shown advantages of training until concentric failure in the maximal strength, ${ }^{6}$ and $6 \mathrm{RM}$ strength gains, ${ }^{5}$ other studies have shown no evidence of a greater magnitude of strength adaptations induced by training until failure. ${ }^{9-11}$ Some studies also reported an advantage of performing submaximal repetitions per set (i.e., not to failure) in the maximal power enhancement. ${ }^{4,10}$ In addition, it has been shown that concurrent endurance and strength training performed with repetitions to failure not only impaired the strength 
performance of elite rowers performing concurrent training, but also the specific endurance adaptations and row performance. ${ }^{9}$ Although some studies have investigated the effects of training to concentric failure in maximal strength and power output, ${ }^{5,7,9-11}$ there are few investigations on the effects of training to concentric failure on muscle hypertrophy. Recently, Sampson and Groeller investigated young men and observed no additional effect of performing repetitions to muscle failure on the elbow flexors' muscle hypertrophy. ${ }^{10}$ However, these authors compared a training group performing repetitions until failure using a controlled velocity ( $2 \mathrm{~s}$ flexion and $2 \mathrm{~s}$ elbow extension) with two groups, one performing submaximal repetitions at maximal velocity during the concentric phase and $2 \mathrm{~s}$ during the eccentric phase; and, another group performing a maximal velocity for both the concentric and eccentric phases. Thus, it is unclear how the different velocity during the concentric phase in those groups performing submaximal repetitions compared with the group performing repetitions maximum have influenced the results of the study, because training velocity could be also a factor that may influence motor unit activation, and possibly muscle hypertrophy. ${ }^{11}$ Interestingly, in this study, even performing significantly greater total training volume, the repetition to failure group did not achieve greater muscle hypertrophy. ${ }^{11}$ Therefore, although the total volume of strength training sessions may be associated with muscle hypertrophy, ${ }^{12}$ the supposed influence of repetitions to failure on muscle hypertrophy and its relationship with total training volume remains to be elucidated. Moreover, to the best of our knowledge, no study has compared the neuromuscular adaptations induced by different strength training protocols to concentric failure in a group composed of only young women. Recently, Ribeiro et al. suggested that the adaptation in maximal strength is influenced by sex, and women may have a better response in upper-body exercises. ${ }^{13}$ In addition, studies indicated that women have a greater capacity to resist fatigue than men. ${ }^{14}$ Therefore, the purpose of this study was to investigate the neuromuscular adaptations induced by strength training with sets performed to concentric failure or not to failure in the elbow flexors of young women. Our hypothesis was that the group performing repetitions to failure would achieve no additional effect on strength performance. Secondly, we hypothesized that the groups performing a greater total volume of repetitions would achieve greater muscle hypertrophy, independent of the performance of repetitions to failure.

\section{Material and Methods}

\section{Experimental Approach to the Problem}

Before the 10 weeks elbow flexor resistance training program, the participants were electronically randomly assigned to one of three groups: 1) repetitions maximum to failure (RF; $n=30$, three sets of repetitions to failure at $70 \%$ of $1 \mathrm{RM})$; 2) repetitions not leading to failure with equalized volume (RNFV; $n=32$, four sets of 7 repetitions at $70 \%$ of $1-\mathrm{RM}$ ); and 3 ) repetitions not to failure (RNF; $n=27$, three sets of 7 repetitions at $70 \%$ of $1 \mathrm{RM})$. The training volume of the repetitions not to failure group with equalized volume was matched with the training volume of the repetitions maximum to failure group. The repetitions not to failure group performed the same number of sets (i.e., three sets) from the RF group, however with submaximal repetitions (i.e., lower volume). Before the training program, the volunteers were tested for their elbow flexor maximal strength (1RM), peak torque at 60 and $180^{\circ} \cdot \mathrm{s}^{-1}$, muscular endurance, and muscle thickness. The peak torque measurements were repeated only after 10 weeks of the training program. All other measurements were repeated after 5 weeks and after the training program ended (i.e., 10 weeks).

\section{Participants}

Eighty-nine physically active young women $(21.9 \pm 3.3$ years, $62.1 \pm 17.1 \mathrm{~kg}, 1.61 \pm 0.12 \mathrm{~m})$ who had not performed regular resistance training in the previous 6 months voluntarily participated in this study. No subjects were under 18 years old. The women were selected at random from the respondents to fliers distributed to the university campus and by word-ofmouth. The participants were instructed to maintain their nutritional habits during the study period. The subjects were properly informed of the study's purpose, procedures, risks and benefits before reading and signing an informed consent form approved by the Institutional Ethics Committee. The study conformed to the principles outlined in the Declaration of Helsinki. The exclusion criteria included any history of cardiovascular disease or orthopedic limitations. The volunteers had to attend $80 \%$ of the training sessions to be included in the final analysis. ${ }^{15}$

\section{Resistance Training Program}

The training sessions were held two days a week. The sets and exercises were separated by a rest interval of approximately 2 minutes ${ }^{16}$. The elbow flexor training was performed using the free-weight bilateral biceps curl exercise with women in a standing position. The participants were divided into three groups: 1) the volunteers from the repetitions maximum to failure group ( $\mathrm{RF}, \mathrm{n}=30$ ) performed 3 sets to concentric failure; 2) the volunteers from the repetition not to failure with equalized volume group ( $\mathrm{RNFV}, \mathrm{n}=32$ ) performed 4 sets of 7 reps; and 3) the volunteers from the repetitions not leading to failure group ( $\mathrm{RNF}, \mathrm{n}=$ 27) performed 3 sets of 7 reps. The training volume from the RNFV group was matched to the training volume from the RF group. The RNF group performed the same number of sets (i.e., three sets) from the RF group, however with submaximal repetitions (i.e., lower volume). We calculated the equal volume between RNFV and RF based on a pilot study with subsample, in 


\section{Strength training with repetitions to failure}

Eur J Transl Myol 27 (2): 113-120

which we verified the number of repetitions when sets are performed until muscle failure. The training load used was $70 \%$ of the 1RM for all groups. Both the training load and the number of sets and repetitions were selected based on the recommendations of the American College of Sports Medicine (ACMS) for beginners and moderately trained practitioners. ${ }^{17}$ The training program was based on a linear periodization. The assigned training loads were gradually increased during the course of the 10-week training period based on the 1RM testing. The resistance exercise choice and order were identical for the three treatment groups. Each volunteer was instructed to record a training log (repetitions performed in each set) for each workout day. In addition to the elbow flexor training sessions, the volunteers performed whole body overall resistance training using exercises that do not involve the elbow flexor muscles (4 - 6 exercises performing sub maximal repetitions). The training sessions were closely supervised by a certified strength and conditioning trainer and an exercise specialist. ${ }^{15}$

\section{Maximal Strength assessment (1RM)}

The elbow flexors' 1RM was assessed before and after the 5 and 10-weeks training periods. The exercise used as test for elbow flexors was the bilateral bicep curl. We determined the highest load that could be lifted in 1 single repetition using a previously published protocol, ${ }^{18}$ with women positioned in a standing position. After performing a specific warm-up (using submaximal loads), the resistance was adjusted to estimate the 1RM. The volunteers were instructed to lift the load once, while maintaining proper technique and completing the full range of motion. Each successive lift was attempted after a 5-minute rest. The load was progressively increased until failure, with no more than 5 attempts performed. The greatest load completed with proper technique was deemed to be the 1-RM. The baseline test and retest intraclass correlation coefficient for the 1-RM test was 0.96 (0.90-0.98).

\section{Isokinetic Peak Torque}

Unilateral elbow flexion peak torque (PT) was tested with 2 sets of 4 concentric repetitions at $60^{\circ} \cdot \mathrm{s}^{-1}$ and 2 sets of 4 concentric repetitions at $180^{\circ} \cdot \mathrm{s}^{-1}$ on a Biodex System 3 isokinetic dynamometer (Biodex Medical, Inc., Shirley, N.Y., USA), with 60 s of rest between sets. The participants were seated on a Scott Bench with their elbow aligned with the axis of rotation of the dynamometer's lever arm. ${ }^{19-21}$ The forearm remained in a supinated position throughout the test. Verbal encouragement was given throughout the test. The test was performed before and after 10 weeks of training. All tests were administered by the same investigator. The ICC values for isokinetic variables ranged from 0.96 to 0.99 .

\section{Muscle endurance}

The muscle endurance was assessed using a repetition to failure test described elsewhere. ${ }^{22}$ Briefly, the test was performed with $70 \%$ of the baseline 1RM before and after the 5 and 10 -week training periods. The participants were instructed to perform the repetitions according to the metronome (1 second for concentric actions and 3 seconds for eccentric actions). ${ }^{23-25}$ The test was concluded when they were unable to maintain the pace of the metronome and/or the total range of motion. The maximum number of repetitions performed was recorded for analysis. The ICC values for muscular endurance was 0.92 .

\section{Muscle thickness}

The muscle thickness (MT) of the participants' elbow flexors was tested before and after the 5 and 10-week training periods. All tests were conducted at the same time of the day, and the participants were instructed to hydrate normally for the $24 \mathrm{~h}$ before the tests. The measurements were taken 5 days after the last training session so that swelling did not affect the MT measurement. ${ }^{26}$ During this time, the participants were instructed to abstain from participating in any other exercise sessions or intense activity. The MT was measured using B-Mode ultrasound (Philips-VMI, Ultra Vision Flip, model BF). A water soluble transmission gel was applied to the measurement site, and a 7.5 MHz ultrasound probe was placed perpendicular to the tissue interface. The MTs of the elbow flexors were measured in accordance with the procedure described by Abe et al. ${ }^{27}$ The muscle thickness of the elbow flexors was considered to be the distance from the subcutaneous adipose tissue-muscle interface to the muscle-bone interface. $^{27}$ The same operator performed all measurements. The baseline test and retest intraclass correlation coefficient for the elbow flexor muscle thickness was 0.98 (0.98-0.99).

\section{Statistical analysis}

The SPSS statistical software package (version 17.0) was used to analyze all data. The results are reported as the mean \pm SD. Normal distribution and homogeneity parameters were checked with Shapiro-Wilk and Levene test's respectively. The training-related effects were assessed using a mixed model two-way Analysis of Variance (ANOVA, group $\mathrm{x}$ time). When a significant $\mathrm{F}$ value was achieved, Bonferroni's post hoc tests were used to determine the pairwise differences between the different time points (1RM and muscle endurance values), and Tukey's post hoc tests were used to determine the pairwise differences between groups. Retrospective statistical power provided by SPSS ranged from 0.85 to 1.00 when a significant time effect was observed, and 0.90 when a significant time vs. group interaction was observed. Significance was accepted at $\mathrm{p}<0.05$. The effect size (ES) between preand post-training for each group was calculated using 


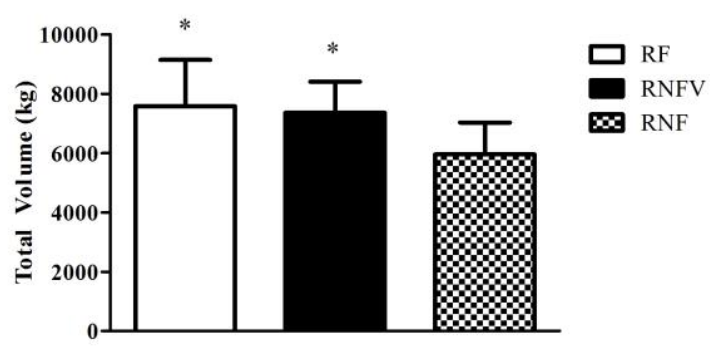

Fig 1. Training volume performed by groups after 10 weeks. *Higher than RNF ( $p<0.05)$. Data are means and $S D . R F$, repetitions to failure. $R N F V$, repetitions not to failure volume equalized. RNF, repetitions not to failure.

Hedges' ES, represented by the following formula: ES = $\left(\mathrm{M}_{\text {post }}-\mathrm{M}_{\text {pre }}\right) / \mathrm{SD}_{\text {pooled }}$, where $\mathrm{M}_{\text {post }}$ is the mean posttraining measure, $\mathrm{M}_{\mathrm{pre}}$ is the mean pre-training measure for each group, and $\mathrm{SD}_{\text {pooled }}$ is the pooled $\mathrm{SD}$ of the pre and post measurements. ${ }^{28}$ The threshold values for assessing the magnitude of the standardized effects, which are the changes as a fraction or multiple of the baseline standard deviation, were $0.20,0.60,1.2$ and 2.0 for small, moderate, large and very large, respectively. ${ }^{29}$

\section{Results}

Before training, there were no differences in the ages, body mass, and height of the RF $(\mathrm{n}=30 ; 22.3 \pm 3.8 \mathrm{yrs}$, $63.7 \pm 22.5 \mathrm{~kg}, 161.6 \pm 6.0 \mathrm{~cm}), \mathrm{RNFV}(\mathrm{n}=32 ; 21.7 \pm$ 2.8 yrs, $60.2 \pm 13.5 \mathrm{~kg}, 162.7 \pm 6.0 \mathrm{~cm})$, and RNF $(\mathrm{n}=$ $27 ; 21.6 \pm 3.3$ yrs, $62,5 \pm 14.1 \mathrm{~kg}, 162.2 \pm 6.2 \mathrm{~cm}$ ) groups. In addition, before training, there were no differences in the muscle thickness, muscle endurance, $1 \mathrm{RM}$ strength and peak torque at $60^{\circ} \cdot \mathrm{s}^{-1}$ and $180^{\circ} \cdot \mathrm{s}^{-1}$ of the RF, RNFV, and RNF groups. Regarding the training regimen, all groups performed the resistance exercise protocol with the same relative $70 \%$ 1RM load. No significant difference was observed in the average load lifted between groups $(p>0.05)$ over the 10 weeks of

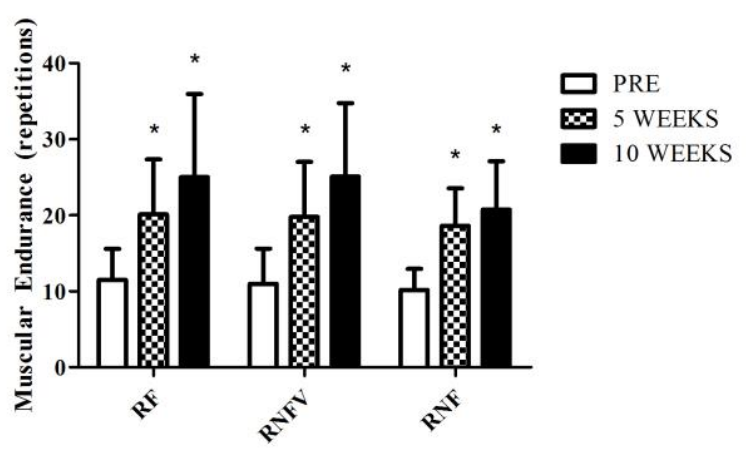

Fig 2. Muscle endurance at baseline (PRE), after 5 and 10 weeks. ${ }^{*}$ Higher than PRE $(p<0.05)$. Data are means and $S D$. $R F$, repetitions to failure. RNFV, repetitions not to failure volume equalized. RNF, repetitions not to failure. training. As expected and in accord with the experimental design, the training volumes performed by the RF and RNFV groups were significantly higher than the RNF group ( $p<0.01$, Figure 1). All data presented normal distribution and homogeneity among groups assessed.

\section{Maximal 1RM strength and isokinetic peak torque}

There were significant increases $(\mathrm{p}<0.001)$ in the $1 \mathrm{RM}$ strength of the RF (ES=0.64 and 0.99 after 5 and 10 weeks, respectively), RNFV (ES=1.00 and 1.26 after 5 and 10 weeks, respectively), and RNF groups (ES=0.76 and 1.00 after 5 and 10 weeks, respectively) after 5 and 10 weeks of training. However, there was no significant time vs. group interaction. In addition, there was a significant time vs. group interaction in the peak torque at $60^{\circ} \cdot \mathrm{s}^{-1}(\mathrm{p}=0.05)$. A significant increase in the peak torque at $60^{\circ} \cdot \mathrm{s}^{-1}$ was observed only in the RNFV group $(E S=0.56, p<0.001)$, and no changes were observed in the RNF (ES = 0.41) and RF groups ( $\mathrm{ES}=0.07)$. Furthermore, there was a significant time vs. group interaction in the peak torque at $180^{\circ} \mathrm{s}^{-1}(\mathrm{p}<0.001)$. Significant increases were observed in the peak torque at $180^{\circ} \cdot \mathrm{s}^{-1}$ in the RNFV $(\mathrm{ES}=0.63, \mathrm{p}<0.001)$ and the RNF groups $(E S=0.19, p<0.01)$, but no change was observed in the RF group (ES $=-0.02$, Table 1 ).

\section{Muscle endurance}

There were significant increases $(p<0.001)$ in the muscle endurance of the RF ( $\mathrm{ES}=1.18$ and 1.25 after 5 and 10 weeks, respectively), the RNFV ( $E S=1.42$ and 1.44 after 5 and 10 weeks, respectively), and the RNF groups (ES=1.16 and 0.82 after 5 and 10 weeks, respectively), after 5 and 10 weeks of training, with no significant time vs. group interaction (Figure 2).

\section{Muscle thickness}

There were significant time effects $(p<0.001)$ and time vs. group interaction $(p<0.01)$ for muscle thickness. There were significant increases in muscle thickness in the RF and the RNFV groups after 5 (RF: $\mathrm{ES}=0.31$,

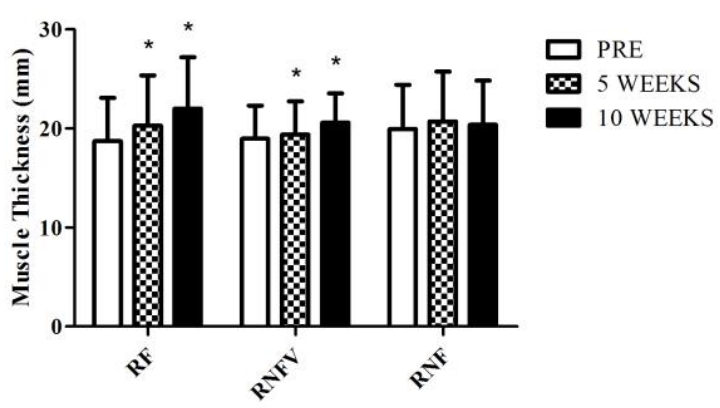

Fig 3. Muscle thickness at baseline (PRE), after 5 and 10 weeks. ${ }^{*}$ Higher than PRE ( $\left.p<0.05\right)$. Data are means and $S D$. RF, repetitions to failure. RNFV, repetitions not to failure volume equalized. $R N F$, repetitions not to failure. 
Table 1. Maximal strength (1RM) and isokinetic peak torque (PT) at $60^{\circ} \cdot \mathrm{s}^{-1}$ and $180^{\circ} \cdot \mathrm{s}^{-1}$ at baseline (PRE), after 5 and 10 weeks (5wk and $10 \mathrm{wk}$, respectively) values

\begin{tabular}{|c|c|c|c|c|c|c|c|c|c|}
\hline & \multicolumn{3}{|c|}{$\mathrm{RF}$} & \multicolumn{3}{|c|}{ RNF } & \multicolumn{3}{|c|}{ RNFV } \\
\hline & PRE & $5 \mathrm{wk}$ & $10 \mathrm{wk}$ & PRE & $5 \mathrm{wk}$ & $10 \mathrm{wk}$ & PRE & $5 \mathrm{wk}$ & $10 \mathrm{wk}$ \\
\hline 1RM (kg) & $17.17 \pm 4.20$ & $19.90 \pm 4.48^{*}$ & $22.03 \pm 4.45^{*}$ & $17.70 \pm 3.87$ & $20.96 \pm 4.05^{*}$ & $22.44 \pm 4.32 *$ & $16.44 \pm 2.95$ & $19.71 \pm 2.62 *$ & $21.09 \pm 2.74^{*}$ \\
\hline PT $60^{\circ} . \mathrm{s}^{-1}$ (N.m) & $26.81 \pm 6.26$ & - & $27.26 \pm 7.01$ & $27.19 \pm 5.87$ & - & $29.63 \pm 5.94$ & $24.57 \pm 4.43$ & - & $27.08 \pm 4.22 *$ \\
\hline PT $180^{\circ} \cdot \mathrm{s}^{-1}$ (N.m) & $21.74 \pm 5.93$ & - & $21.63 \pm 6.36$ & $21.88 \pm 5.21$ & - & $22.79 \pm 4.45^{*}$ & $19.06 \pm 3.51$ & - & $21.68 \pm 4.33 *$ \\
\hline
\end{tabular}

* Higher than PRE (p<0.05). Data are means and SD. RF, repetitions to failure. RNFV, repetitions not to failure volume equalized. $R N F$, repetitions not to failure.

and RNFV: $0.13 \mathrm{p}<0.001)$ and 10 weeks of training (RF: 0.57 , and RNFV: $0.50, \mathrm{P}<0.001$ ), but significant changes were not observed in the RNF subjects ( $E S=0.20$ and 0.09 after 5 and 10 weeks, respectively, NS, Figure 3).

\section{Discussion}

The main findings of the present study were that strength training performed with repetitions to failure does not induce additional gains in the maximal 1RM strength, muscle endurance or muscle hypertrophy of the elbow flexors of young women. Another interesting result was that repetitions to failure may even impair the force produced at faster muscle action velocities. In addition, independent of the maximum or submaximal training routine, the groups that performed the higher training volume (i.e., RF and RNFV) presented the higher levels of muscle thickness gains when compared to the repetitions submaximal not to failure group (i.e., RNF). To date, few studies have investigated the effects of training to failure on strength gains. In a study by Rooney et al., forty-two young subjects (18 males and 24 females) performed 6 weeks of strength training with repetition to failure sets (i.e., 6RM) versus not to failure sets (i.e., subjects resting 30 seconds between each repetition during the sets with similar volume and overload). ${ }^{7}$ These authors observed a significantly greater magnitude of strength gains in the RM group compared to the submaximal group (56.3 vs. $41.2 \%$ ). Similarly, Drinkwater et al. observed greater 6 RM strength gains in junior sports athletes performing 4 sets of 6RM compared with subjects performing 8 sets of 3 repetitions with loads of $6 \mathrm{RM}{ }^{5}$ However, these authors actually tested 6RM, and the maximal dynamic strength (i.e., 1RM) was not assessed in their study. In the present study, besides there were no differences in 1RM strength gains among groups, there was a significant increase in the peak torque at $60 \mathrm{~s}^{-1}$ only in the RNFV group. Regarding the $1 \mathrm{RM}$, our results are in agreement with Folland et al. ${ }^{9}$ who compared two strength training protocols with high and low fatigue after nine weeks and did not find any difference between groups in the strength gains. In addition, our results are also in agreement with Izquierdo et al. who investigated young active men performing strength training with sets performed until concentric failure and subjects performing repetitions not to failure during 16 weeks, and found similar strength adaptations in the squat and bench press exercises between training groups. ${ }^{10}$ More recently, Sampson and Groeller investigated 28 young men before and after 12 weeks of strength training and did not observe any influence of repetition maximum performance in the maximal strength gains of the elbow flexors $(28.6-32.8 \%){ }^{11}$ In this study, one group performed repetitions until muscle failure (6RM), and the others performed 4 submaximal repetitions at the same load (one group performed explosive concentric and eccentric muscle actions, and the other group performed only explosive concentric actions). To the best of our knowledge, the present study is the first study investigating the effects of strength training with repetitions until muscle failure in a group of only young women, and our results suggest that the submaximal repetition regimen is an optimal stimulus to promote strength gains in the elbow flexors in this population, even when a low volume of repetitions is performed (i.e., RNF subjects). Our results are in agreement with those of Sampson and Groeller, who showed the same strength gains even in the groups who performed a lower total volume (submaximal repetitions group). ${ }^{11}$ It is unclear why only the RNFV group improved the peak torque at $60 \mathrm{~s}^{-1}$, but this could be related to the greater magnitude of the effect observed in the 1 the submaximal repetition regimen this group, which may induce transference to a non-specific strength test (i.e., the isokinetic test) in this group. However, this explanation remains speculative and needs to be further investigated. Surprisingly, there were no differences in the muscle endurance between groups in the present study. It could be suggested that performing repetitions to concentric failure may be necessary to stimulate the capacity to tolerate muscle fatigue. Indeed, it has been shown that the metabolic disturbance and fatigue are higher when performing the maximum repetitions compared to submaximal repetitions in lower limb exercises. $^{3,4,8}$ In addition, Izquierdo and colleagues have shown that repetitions until failure induced greater muscle endurance gains in the bench press exercise in athletes, ${ }^{10}$ but the same was not observed in the squat exercise because the maximal and submaximal groups 
achieved the same muscle endurance gains. They also argued that the lower extremities in these elite athletes were highly conditioned, which could explain their results. ${ }^{10}$ Thus, the added effects of training to failure were not evident. In addition, the advantage of training to failure for the upper-body musculature may have provided a novel stimulus and may have further enhanced muscle endurance. However, this result was not observed in the upper-body endurance of the women in the present study. Thus, taking into consideration our results and those by Izquierdo and colleagues, ${ }^{10}$ one could suggest that the influence of performing repetitions until muscle failure on muscle endurance gains might be dependent on the muscle group trained, training status, and gender. However, these differences also require further investigation. In the present study, only those groups who performed submaximal repetitions increased the peak torque at $180^{\circ} \cdot \mathrm{s}^{-1}$. Our results are in accord with Izquierdo and colleagues ${ }^{10}$, who demonstrated greater skeletal muscle power gains in the squat exercise in the group that performed repetitions not to failure compared to the repetitions to failure group, although these authors did not observe between-group differences in the power output gains in the bench press exercise. A possible mechanistic explanation for the lack of increase in the peak torque at $180^{\circ} \cdot \mathrm{s}^{-1}$ in the RF group may be related to the decrease in the muscle action velocity during the last few repetitions of the training set and subsequent sets. In fact, the muscle power output gains are largely reduced when strength training is performed with repetitions until muscle failure. ${ }^{8}$ In contrast to the maximal strength and muscle endurance results, the groups in the present study that performed a greater total training volume (RF and RNFV) induced greater elbow flexor muscle hypertrophy, which was not observed in the RNF group. Indeed, it has been recently shown that muscle thickness adaptations are positively associated with the total work performed during concentric training. ${ }^{12}$ Nevertheless, muscle fatigue did not provide any additional stimulus to muscle hypertrophy because similar muscle thickness gains were observed in the RF and RNFV groups. These results are in agreement with Sampson and Groeller who did not observe an additional effect of performing repetitions until muscle failure in elbow flexor muscle hypertrophy in young men. ${ }^{11}$ However, these authors observed the same muscle hypertrophy, even in the groups who performed a lower amount of total work. The possible discrepancies between these studies could be explained by the different training protocols used and the different populations studied (i.e., men versus women). In fact, it seems that this is the first study to investigate the muscle hypertrophy induced by repetitions maximal and submaximal in a group of only women, and, based on our results, we can suggest that repetitions until muscle failure are not necessary to optimize muscle hypertrophy when the total volume of repetitions is equalized. In addition, based on the present results, it is important to mention that the repetitions to failure regime may impair the neuromuscular outcomes related to fast force production such as power, rate of force development, and rate of velocity development. Another negative effect of performing sets until concentric failure is the decrease in muscle recovery due to the higher metabolic impact ${ }^{8}$. Therefore, the goals and risks of resistance training until to concentric failure must be considered before its prescription. It is important to observe that the present study assessed the effects of three different strength training protocols in active women who had not received previous resistance training, which limits the extrapolation of our results to well-trained young women. In addition, we only assessed the elbow flexors, which limits the applicability of our results to other muscle groups. Moreover, we only assessed our subjects after 5 and 10 weeks of training, and it remains unclear whether the differences or similarities observed between the training protocols would be maintained over longer periods. On the other hand, the investigation of training adaptations in the elbow flexors of previously untrained young women has the advantage of assessing a muscle group that is usually not well developed in women. Furthermore, the absence of additional stimuli from the repetitions to failure during strength training is well documented in other muscle groups, ${ }^{3,4,10}$ although data on the influence of repetitions until failure in muscle hypertrophy are limited.

In summary, similar elbow flexor 1RM strength and muscle endurance improvements were observed in young women after 10 weeks of training, independent of the performance of the repetitions until failure or training volume. Moreover, higher force production was observed when submaximal repetitions were performed and the total volume was equalized by an additional set (i.e., RNFV). On the other hand, only those groups who performed a greater total volume of repetitions (RF and RNFV) increased their muscle thickness. This result suggests that the subjects elbow flexor muscle hypertrophy may be optimized using a greater volume of total work, whereas muscle fatigue (i.e., repetitions until failure) does not add any additional stimulus. From a practical standpoint, repetitions until failure are not necessary to optimize the enhanced muscle strength and endurance induced by strength training, and may even impair strength development at higher velocities, as observed in the PT $180^{\circ} . \mathrm{s}^{-1}$ results. It seems that there is a stimulus threshold in which training with more repetitions does not provide further strength gains. On the other hand, within the volumes investigated, it seems that a greater volume of total work may be a necessary to achieve muscle hypertrophy in previously untrained women. Therefore, our results suggest that submaximal repetitions without reducing the total work (i.e., adding more sets) seems to be the best strategy to induce overall neuromuscular adaptations in young women. Training until concentric failure should be 
further investigated, including studying their effects in longer training periods and other periodization models.

\section{List of acronyms}

$\mathrm{RF}$ - repetitions to failure

RNFV - repetitions not to failure with equalized volume

RNF - repetitions not to failure

1RM - one repetition maximum

PT - peak torque

MT - muscle thickness

SD - standard deviation.

\section{Author's contributions}

SM accountable for all aspects of the work, like conception, design and drafting of the work, the acquisition, analysis and interpretation of data.; EC: scientific reviewer and co-advisor; Mikel Izquierdo: scientific and translate reviewer; RC: acquisition data; AM: acquisition data; VA: acquisition data; JA: acquisition data; MB: advisor and final approval of the version to be published.

\section{Acknowledgments}

This study was partially supported by the Council for the Research Development $(\mathrm{CNPq})$ and by the Coordination for the Improvement of Higher Level Personnel (Capes).

\section{Conflict of Interest}

The authors declare that there are no conflicts of interest regarding this manuscript. They also declare that they have not received any funding for this study.

\section{Corresponding Author}

Saulo Santos Martorelli University of Brasilia, College of Physical Education, Campus Universitário Darcy Ribeiro, Brasília-DF, 70.919-970, Brazil.

Phone: +55 $613107-2526$

E-mail: martorelli.saulo@gmail.com

\section{E-mails of coAuthors}

Eduardo Cadore: edcadore@yahoo.com.br ;

Mikel Izquierdo: mikel.isquierdo@gmail.com ;

Rodrigo Celes: celes.rodrigo@gmail.com ;

André Martorelli: andremartorelli@gmail.com ;

Vitor Alonso: alonsovitor@gmail.com ;

José Alvarenga: jgsama@unb.br ;

Martim Bottaro: martim@unb.br .

\section{References}

1. Steele J, Fisher J, Giessing J, Gentil P. Clarity in reporting terminology and definitions of set end points in resistance training. Muscle Nerve 2017;Jan 3. doi: 10.1002/mus.25557.

2. Izquierdo M, Gonzalez-Badillo JJ, Hakkinen K, et al. Effect of loading on unintentional lifting velocity declines during single sets of repetitions to failure during upper and lower extremity muscle actions. Int J Sports Med 2006;27:718-24.
3. Izquierdo M, Gonzalez-Izal M, NavarroAmezqueta I, et al. Effects of strength training on muscle fatigue mapping from surface emg and blood metabolites. Med Sci Sports Exerc 2011;43:303-11.

4. Izquierdo $\mathrm{M}$, Ibanez $\mathrm{J}$, Calbet $\mathrm{JA}$, et al. Neuromuscular fatigue after resistance training. Int J Sports Med 2009;30:614-23.

5. Drinkwater EJ, Lawton TW, Lindsell RP, et al. Training leading to repetition failure enhances bench press strength gains in elite junior athletes. $\mathbf{J}$ Strength Cond Res 2005;19:382-8.

6. Jenkins ND, Housh TJ, Bergstrom HC, et al. Muscle activation during three sets to failure at 80 vs. $30 \% 1 \mathrm{RM}$ resistance exercise. Eur J Appl Physiol 2015;115:2335-47.

7. Rooney KJ, Herbert RDBalnave RJ. Fatigue contributes to the strength training stimulus. Med Sci Sports Exerc 1994;26:1160-4.

8. Gorostiaga EM, Navarro-Amezqueta I, Calbet JA, et al. Energy metabolism during repeated sets of leg press exercise leading to failure or not. PLoS One 2012;7:e40621.

9. Folland JP, Irish CS, Roberts JC, et al. Fatigue is not a necessary stimulus for strength gains during resistance training. Br J Sports Med 2002;36:3703; discussion 374 .

10. Izquierdo M, Ibanez J, Gonzalez-Badillo JJ, et al. Differential effects of strength training leading to failure versus not to failure on hormonal responses, strength, and muscle power gains. J Appl Physiol 2006;100:1647-56.

11. Sampson JA, Groeller H. Is repetition failure critical for the development of muscle hypertrophy and strength? Scand J Med Sci Sports 2016;26:375-83. doi: 10.1111/sms.12445. Epub 2015 Mar 24.

12. Cadore EL, Pinto RS, Bottaro MIzquierdo M. Strength and endurance training prescription in healthy and frail elderly. Aging Dis 2014;5:18395.

13. Ribeiro AS, Avelar A, Schoenfeld BJ, et al. Effect of 16 weeks of resistance training on fatigue resistance in men and women. $\mathrm{J}$ Hum Kinet 2014;42:165-74.

14. Hunter SK. Sex differences and mechanisms of task-specific muscle fatigue. Exerc Sport Sci Rev 2009;37:113-22. doi: 10.1097/JES.0b013e3181aa 63e2. Review.

15. Gentil PBottaro M. Influence of supervision ratio on muscle adaptations to resistance training in nontrained subjects. J Strength Cond Res 2010;24:639-43.

16. Celes R, Brown LE, Pereira MC, et al. Gender muscle recovery during isokinetic exercise. Int $\mathrm{J}$ Sports Med 2010;31:866-9.

17. ACSM. American college of sports medicine position stand. Progression models in resistance 


\section{Strength training with repetitions to failure}

Eur J Transl Myol 27 (2): 113-120

training for healthy adults. Med Sci Sports Exerc 2009; 41: 687-708.

18. Brown LEWeir JP. Asep procedures recommendation i: Accurate assessment of muscular strength and power. Journal of Exercise Physiology - online 2001;4:1-21.

19. Flores DF, Gentil P, Brown LE, et al. Dissociated time course of recovery between genders after resistance exercise. J Strength Cond Res 2011;25:3039-44.

20. Gentil P, Soares SR, Pereira MC, et al. Effect of adding single-joint exercises to a multi-joint exercise resistance-training program on strength and hypertrophy in untrained subjects. Appl Physiol Nutr Metab 2013;38:341-4.

21. Pereira M, Bottaro M, Brown L, et al. The effects of graduated compression sleeves on muscle performance: A randomised controlled trial. International Journal of Sports Science and Coaching 2014; 9: 985-992.

22. Martorelli SS, Martorelli AS, Pereira MC, et al. Graduated compression sleeves: Effects on metabolic removal and neuromuscular performance. J Strength Cond Res 2015; 29: 1273 8.

23. Gonzalez-Badillo JJSanchez-Medina L. Movement velocity as a measure of loading intensity in resistance training. Int J Sports Med 2010;31:34752.

24. Hatfield DL, Kraemer WJ, Spiering BA, et al. The impact of velocity of movement on performance factors in resistance exercise. J Strength Cond Res 2006;20:760-6.

25. Sakamoto ASinclair PJ. Effect of movement velocity on the relationship between training load and the number of repetitions of bench press. J Strength Cond Res 2006;20:523-7.

26. Chilibeck PD, Stride D, Farthing JPBurke DG. Effect of creatine ingestion after exercise on muscle thickness in males and females. Med Sci Sports Exerc 2004;36:1781-8.

27. Abe T, Dehoyos DV, Pollock MLGarzarella L. Time course for strength and muscle thickness changes following upper and lower body resistance training in men and women. Eur J Appl Physiol 2000;81:174-80.

28. Hedges L, Olkin I. Statistical methods for metaanalysis. 1985: Academic Press.

29. Hopkins WG, Marshall SW, Batterham AMHanin J. Progressive statistics for studies in sports medicine and exercise science. Med Sci Sports Exerc 2009;41:3-13. 